\title{
Detergent-free functionalisation of hybrid vesicles with membrane proteins using SMALPs
}

\author{
Rosa Catania, ${ }^{[a, b]}$ Jonathan Machin, ${ }^{[a, b]}$ Michael Rappolt, ${ }^{[c]}$ Stephen P. Muench, ${ }^{[a, b]}$ Paul A. \\ Beales, ${ }^{*[a, d]}$ and Lars J.C. Jeuken $*[a, b, e]$ \\ ${ }^{a}$ Astbury Centre of Structural Molecular Biology, University of Leeds, LS2 9JT, Leeds, United Kingdom \\ ${ }^{\mathrm{b}}$ School of Biomedical Sciences, University of Leeds, LS2 9JT, Leeds, United Kingdom \\ ' School of Food Science and Nutrition, University of Leeds, LS2 9JT, Leeds, United Kingdom \\ ${ }^{d}$ School of Chemistry, University of Leeds, LS2 9JT, Leeds, United Kingdom \\ e Leiden Institute of Chemistry, Leiden University, PO Box 9502, 2300 RA, Leiden, the Netherlands \\ * Corresponding authors: P.A.Beales@leeds.ac.uk, L.J.C.Jeuken@lic.leidenuniv.nl
}

\begin{abstract}
:
Hybrid vesicles (HVs) that consist of mixtures of block copolymers and lipids are robust biomimetics of liposomes, providing a valuable building block in bionanotechnology, catalysis and synthetic biology. However, functionalisation of HVs remains laborious and expensive, creating a significant current challenge in the field. Here, using a new approach of extraction with styrene-maleic acid lipid particles (SMALPs), we show that a membrane protein (cytochrome bo $_{3}$ ) directly transfers into HVs with an efficiency of $73.9 \pm 13.5 \%$ and without the requirement of any detergent, long incubation times or mechanical disruption. Interestingly, direct transfer of membrane proteins using this approach was not possible into liposomes. This suggests that the HVs are more amenable than liposomes to membrane protein incorporation from a SMALP system. Finally, we show that this transfer method is not limited to cytochrome $b_{3}$ and can also be performed with complex membrane protein mixtures.
\end{abstract}


Vesicles made of natural or synthetic lipids (liposomes) are a suitable platform for mimicking structures and functions found in nature. ${ }^{[1,2]}$ Liposomes have been widely exploited to fabricate artificial compartments in bottom-up synthetic biology (artificial cells and organelles) and nanoreactors in compartmentalised (photo)catalysis. ${ }^{[3,4]}$ Functionalisation of liposomes in biotechnology is achieved by the reconstitution of membrane proteins (MPs), which in spite of their complex amphiphilic nature, have an increasing number of promising applications in areas such as drug discovery, ${ }^{[5]}$ vaccines, $^{[6]}$ biosensors ${ }^{[7]}$ and energy conversion. ${ }^{[8]}$ However, the application of proteoliposomes is still hampered by the lack of chemical and physical long-term stability (typically days ${ }^{[9]}$ and the complexity of extraction and reconstitution of MPs. ${ }^{[10,11]}$

Recent developments using amphiphilic polymers have shown promise in solving these experimental limitations. Amphiphilic polymers can self-assemble into robust and stable vesicles, known as polymersomes. ${ }^{[12,13]}$ Despite the advantageous stability and tunability of these synthetic vesicles, ${ }^{[14]}$ the non-native polymeric environment can limit the functional incorporation of many MPs. ${ }^{[15]}$ Hybrid vesicles (HVs), composed of a mixture of block copolymers and lipids, have proven to be a balanced compromise between liposome biocompatibility and polymersome stability. ${ }^{[16-19]}$ Several block copolymers have been studied to correlate how their chemical structure affects the overall properties of the hybrid vesicles, and both well-mixed vs phase-separated membranes have been used. ${ }^{[15,20,21]}$ We have previously shown that the membrane protein cytochrome bo $\left(c y t b o_{3}\right.$ ) can be functionally reconstituted into hybrid vesicles containing up to $50 \mathrm{~mol} \%$ of the diblock copolymer poly(butadiene- $b$-ethylene oxide) $\left(\mathrm{PBd}_{22}-b-\mathrm{PEO}_{14}\right)$ with POPC lipids, with minimal loss in protein activity and enhanced lifetime up to 500 days. ${ }^{[16,22]}$

Despite the promise of polymersomes and HVs, the process of extraction, isolation and functional reconstitution of MPs still presents major challenges. Reconstitution methods into polymersomes and HVs are based on methods developed for reconstitution in liposomes, which require detergents and often extensive optimisation. Detergents can destabilise MPs by inducing protein unfolding, dissociation of small subunits, removal of natural lipids associated with the protein hydrophobic regions, and consequently compromise their activity and limit their functional lifetime. ${ }^{[23-25]}$ Thus, the selection of a compatible detergent and optimum condition to extract a target protein can be a laborious, time-consuming and riskprone procedure. ${ }^{[26,27]}$ 
Here, we report a novel strategy for the reconstitution of a model membrane protein, cyt bo 3 from Escherichia coli (Figure 1A), into HVs that does not require the use of a detergent. Transfer of $\mathrm{cyt}_{\mathrm{b}} \mathrm{bo}_{3}$ is instead accomplished by a second amphiphilic polymer, styrene-maleic acid copolymer (SMA, Figure 1B). SMA and similar polymers have emerged as an effective material to extract and solubilise MPs while preserving protein activity, ${ }^{[28]}$ overcoming issues encountered with detergent-mediated solubilisation. ${ }^{[29,30]}$ SMA are anionic copolymers containing carboxylic acid pendant groups in the form of maleic acid alternating with the hydrophobic styrene pendant groups (Figure 1B). Unlike detergents, SMA copolymers do not self-assemble into micelles. ${ }^{[31]}$ When added to cellular membrane extracts, the hydrophobic styrene groups of SMA copolymers intercalate between the acyl chains of the lipid bilayer, whereas the hydrophilic maleic acid groups interface with the solvent. ${ }^{[28]}$ This interaction between SMA copolymers and membranes leads to the spontaneous formation of discoidal particles of $\sim 10 \mathrm{~nm}$ diameter. ${ }^{[32]}$ SMA copolymers offer the advantage of solubilising MPs directly from the cell membrane by forming these nanodisc structures, called SMA-lipid particles (SMALPs), which retain the natural lipids associated with the MPs. ${ }^{[33,34]}$ SMALPs can be purified by affinity chromatography and isolated. ${ }^{[35]}$ Besides their use for MPs structural and functional studies, ${ }^{[35]}$ SMALPs have recently been shown to mediate MPs reconstitution into planar lipid bilayers, as the tetrameric $\mathrm{K}^{+}$channel, ${ }^{[36]}$ and into liposomes, as exemplified for the membrane proteins cytochrome $c$ oxidase ${ }^{[37]}$ and the plasma membrane $\mathrm{Na}^{+} / \mathrm{H}^{+}$ antiporter. ${ }^{[38]}$ In addition to SMA, other maleic acid copolymers capable of solubilising MPs have been synthesised with various chemical functionalities, such as aliphatic side chains replacing the styrene group [39-41] or differently charged moieties in the maleic group, providing a diverse toolkit of potential polymers. ${ }^{[41-43]}$

Cyt $b_{3}$ is a 4 sub-unit membrane enzyme complex ( $\left.143 \mathrm{kDa}\right)$ from $E$. coli that belongs to the heme-copper oxidase enzyme family and, as such, accepts electrons from ubiquinol and passes them onto molecular oxygen, coupling the electron transfer with proton pumping across the membrane (Figure $1 \mathrm{~A}$ ). ${ }^{[44]}$ Activity of $c y t b o_{3}$, and thus functional reconstitution into the membrane vesicles, is commonly evaluated by measuring oxygen consumption. For the hybrid vesicles, we selected $\mathrm{PBd}_{22}-b-\mathrm{PEO}_{14}(\mathrm{MW} 1.8 \mathrm{kDa}$ ) (Figure 1B) as this copolymer is a compromise between the stability of higher MW polymers and minimising the difference in 
hydrophobic thickness between the membranes of pure polymer and pure lipid systems, and forms a homogeneous blend with lipids. ${ }^{[15,45]}$

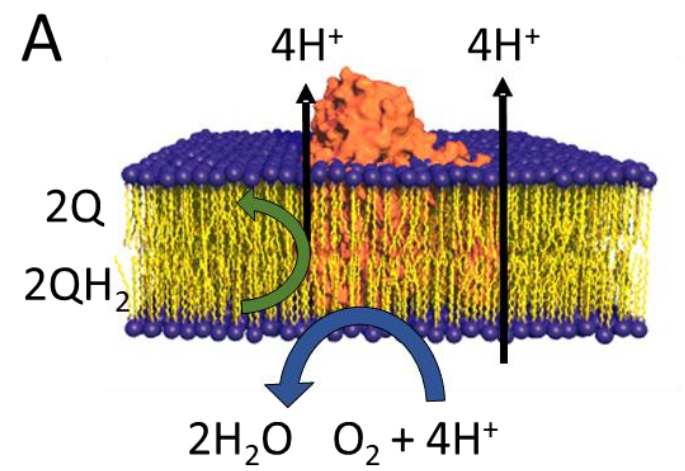

B

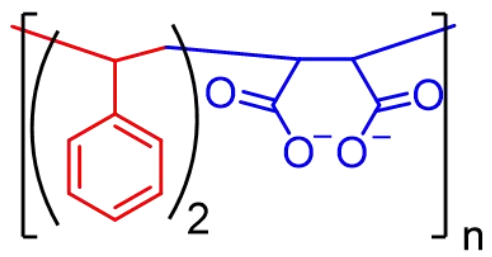

\section{Styrene-maleic acid copolymer}

(SMA)<smiles>C=CC(CC)(CC(C)(C)C)CC(C)(C)O</smiles>

\section{Poly(butadiene- $b$-ethylene oxide) \\ $\left(\mathbf{P B d}_{22}-b-\mathrm{PEO}_{14}\right)$}

Figure 1. (A) Schematic representation of the structure and function of cytochrome $\mathrm{bo}_{3}$ (orange) embedded in the lipid bilayer (represented with yellow lipid tails and blue head groups). (B) Chemical structures of SMA (2:1) copolymer, with in red the styrene group and in blue the maleic acid group, and $\mathrm{PBd}_{22}-b-\mathrm{PEO}_{14}$ copolymer, with in red the polybutadiene block polymer and in green the polyethylene glycol block polymer.

Membrane fractions (protein content $\sim 4 \mathrm{mg} / \mathrm{mL}$ ) from E. coli G0105/pJRhisA, containing Histagged cyt $\mathrm{bo}_{3}$, were incubated with $2 \%(\mathrm{w} / \mathrm{v}) \mathrm{SMA}$ for $2 \mathrm{~h}$ at RT and purified via Ni-NTA affinity chromatography (as described in the Supporting Information). Purification of SMA-solubilised cyt $\mathrm{bo}_{3}$ (SMA-cyt $\mathrm{bo}_{3}$ ) was confirmed in a direct comparison with a previous published procedure $^{[46]}$ using n-Dodecyl- $\beta$-D-maltoside (DDM) solubilisation (Figure S1).

SMA-cyt $b_{3}$ and DDM-cyt $b o_{3}$ were reconstituted into HVs composed of a mixture (1:1 $\mathrm{mol} / \mathrm{mol}$ ratio) of $\mathrm{PBd}_{22}-b-\mathrm{PEO}_{14}$ and E. coli 'polar' lipid extracts. Yield and activity were 
compared with an established reconstitution procedure into pure liposomes (E. coli 'polar' lipid extract). First, we investigated the stability of HVs when exposed to increasing concentrations of SMA copolymer (Figure S2 and S3). SMA is seen to solubilise the HVs at a SMA to lipid and $\mathrm{PBd}_{22}-b-\mathrm{PEO}_{14}$ copolymer ratio of $1(\mathrm{~mol} / \mathrm{mol})$, with less SMA needed to solubilise HVs than liposomes. Still, the amount of SMA required to reconstitute cyt $b_{3}$ is about 20 time less (see below), and thus we excluded that the presence of SMA during the reconstitution of cyt $b_{3}$ could affect the stability of the hybrid vesicles.

To reconstitute cyt $b_{3}$ in HVs, we took advantage of SMALPs precipitating in the presence of $\mathrm{MgCl}_{2}(>5 \mathrm{mM})$ due to the interactions of the divalent cation $\mathrm{Mg}^{2+}$ with the maleic acid groups ${ }^{[47]}$, which has be used to exchange between SMALP into an amphipol scaffold. SMAcyt $\mathrm{bo}_{3}$ was incubated with $\mathrm{HVs}$ (or liposomes as control) on ice for $30 \mathrm{~min}$ at a protein to lipids ratio of $\sim 1: 100(\mathrm{w} / \mathrm{w})$. SMA-cyt $b_{3}$ that was not reconstituted into HVs was removed by centrifugation at $17,000 \mathrm{~g}$ for $15 \mathrm{~min}$ after incubating the mixture with $10 \mathrm{mM} \mathrm{MgCl}$. The supernatant, containing only the HVs, was compared with control samples in which cyt bo3 (purified in DDM) was reconstituted into HVs and liposomes after destabilisation with detergent (Triton X-100), followed by extensive removal of the detergent by Biobeads, as previously reported $^{[16]}$ (described in the Supporting Information).

Dynamic light scattering (DLS) analysis of the four reconstituted samples showed that the diameter of the HVs (Figure 2A) slightly increased after SMAs-cyt bo $o_{3}$ reconstitution (from $130 \mathrm{~nm}$ to $\sim 150 \mathrm{~nm}$ ), while it is reduced following DDM-cyt bos reconstitution ( $100 \mathrm{~nm}$ ). For the liposomes (Figure 2B), the diameter of the vesicles is reduced by DDM-cyt bo reconstitution (from $\sim 140 \mathrm{~nm}$ to $\sim 115 \mathrm{~nm}$ ), suggesting that liposomes either loose lipids or reorganise. This decrease in size is not observed (or to a lesser degree) when reconstituting from SMAs-cyt bo $o_{3}$ samples ( $\left.130 \mathrm{~nm}\right)$. 
A

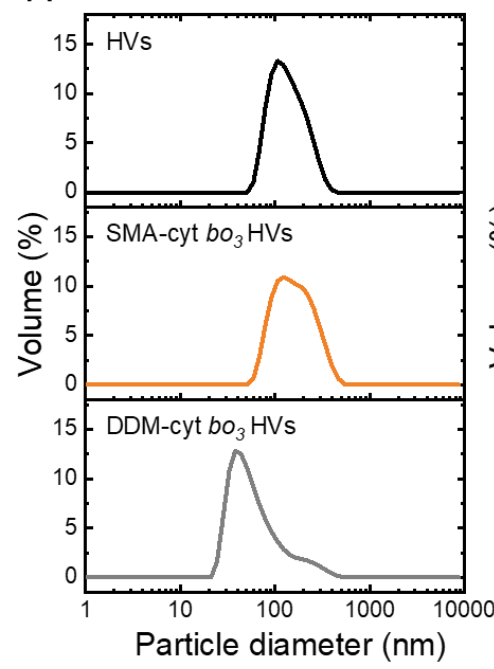

B

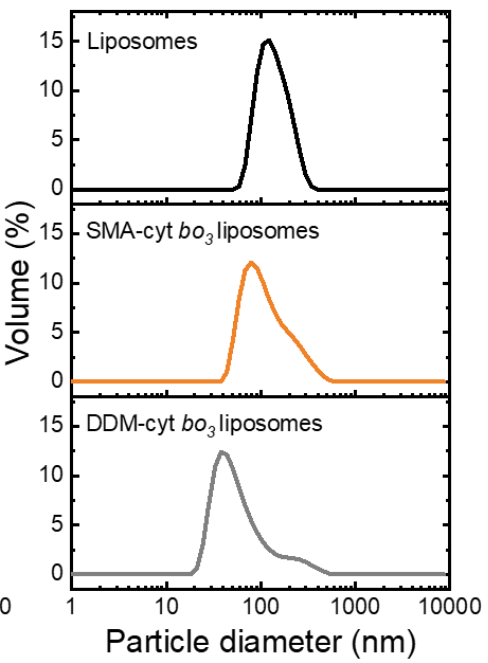

Figure 2 Physical characterisation of membrane vesicles. Dynamic light scattering (DLS) volume profiles of: (A) HVs, SMA-cyt $b o_{3}$ and DDM-cyt $b o_{3}$ reconstituted in HVs (SMA-cyt $b o_{3} \mathrm{HVs}$ and DDMcyt $b o_{3} \mathrm{HVs}$, respectively) and (B) liposomes, SMA-cyt $b o_{3}$ and DDM-cyt bo $o_{3}$ reconstituted in liposomes (SMA-cyt $b o_{3}$ liposomes and DDM-cyt bo 3 liposomes, respectively).

The reconstitution efficiency of cyt $\mathrm{bo}_{3}$ was quantified by solubilisation of the vesicles with Triton X-100 solution and UV-analysis of the Soret peak of cyt bo $(409 \mathrm{~nm})$. Interestingly, the reconstitution efficiency of SMA-cyt $b_{3}$ was profoundly different between $\mathrm{HVs}$ and liposomes (Table 1). SMA-cyt $b_{3}$ could be directly reconstituted into HVs but not into liposomes. This difference in reconstitution efficiency between HVs and liposomes was also confirmed by sodium dodecyl sulphate polyacrylamide gel electrophoresis (SDS-PAGE) (Figure 3). We note that this is the first time that reconstitution of an SMA-solubilised protein has been shown in vesicles without extensive incubation periods or the application of mechanical force. For cytochrome $c$ oxidase, sonication or extrusion were required to induce its reconstitution into liposomes; ${ }^{[37]}$ while for plasma membrane $\mathrm{Na}^{+} / \mathrm{H}^{+}$antiporter, a much longer incubation time (overnight) with liposomes of larger diameter $(400 \mathrm{~nm})$ was needed and only $~ 10 \%$ reconstitution was achieved. ${ }^{[38]}$ In contrast, here we show that a simple incubation for $30 \mathrm{~min}$ on ice is sufficient to reconstitute SMA cyt $b_{3}$ into $\mathrm{HVs}$, while the same procedure does not lead to a transfer of cyt $b o_{3}$ to liposomes. 
Table 1 Reconstitution efficiency of SMA-cyt $b o_{3}$ and DDM-cyt $b o_{3}$ in vesicles as quantified by UV-vis spectroscopy of the Soret band ( $409 \mathrm{~nm}$ ). SMA-cyt $b_{3}$ was reconstituted by simple incubation with $\mathrm{HVs}$ or liposomes. DDM-cyt $\mathrm{bo}_{3}$ was reconstituted after destabilising the HVs or liposomes with Triton $\mathrm{X}-100^{[16]}$ (see Supporting Information for more details).

Solubilised cyt $\mathrm{bo}_{3} \quad$ Membrane vesicles Reconstitution Efficiency (\%) \pm S.D.

\begin{tabular}{llcc}
\hline $\mathrm{SMA}^{-c y t} b o_{3}$ & $\mathrm{HVs}$ & 73.9 & \pm 13.5 \\
$\mathrm{SMA}-\mathrm{cyt} b o_{3}$ & Liposomes & not detected & $(<1)$ \\
DDM-cyt $b o_{3}$ & $\mathrm{HVs}$ & 61.0 & \pm 7.5 \\
DDM-cyt $b o_{3}$ & Liposomes & 58.0 & \pm 3.5 \\
\hline
\end{tabular}

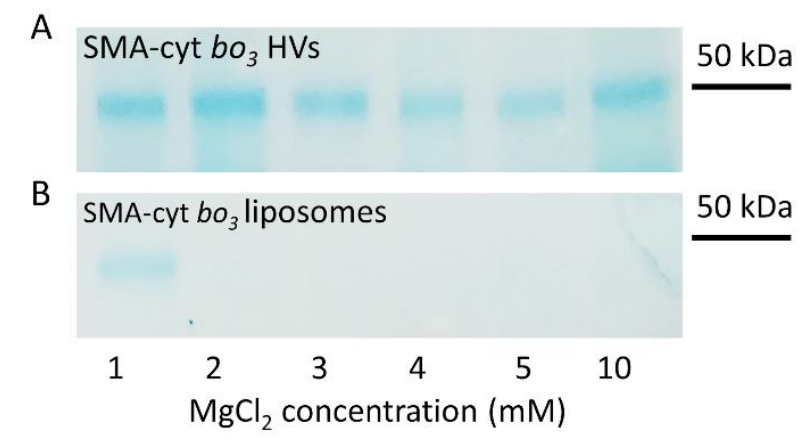

Figure 3 Analysis of reconstituted cyt $b_{3}$ in (A) HVs or (B) liposomes. After direct incubation of SMAcyt $b_{o_{3}}$ with $\mathrm{HVs}$ or liposomes, samples were incubated with increasing $\mathrm{Mg}^{2+}$ concentration for $2 \mathrm{~h}$, followed by centrifugation at $17,000 \mathrm{~g}$ for $15 \mathrm{~min}$ to pellet non-reconstituted SMA-cyt $\mathrm{bo}_{3}$. The supernatant containing HVs or liposomes were analysed with SDS-PAGE (Coomassie Blue staining).

The activities of soluble and reconstituted cyt $b_{3}$ were compared by measuring the rates of oxygen consumption with the substrate ubiquinol $1\left(Q_{1}\right)(200 \mu \mathrm{M})$, which is reduced by dithiothreitol (DTT) (2mM) (Figure 4A, see Supporting Information for details). Figure 4B shows the activity of SMA-cyt $b_{3}$ in the soluble form and after reconstitution into either HVs or liposomes. The activity is compared with $\mathrm{DDM}-\mathrm{cyt} \mathrm{bo}_{3}$ and its reconstitution via previously published methods. ${ }^{[16,22]}$ The activity of the soluble SMA-cyt $b o_{3}$ is significantly lower than the activity of DDM-cyt $b_{3}$. A reduction in activity has been previously reported for other enzymes in SMALPs. ${ }^{[48]}$ A reduction in activity is also apparent after DDM-cyt $b_{3}$ is reconstituted into liposomes. For $c y t b_{3}$, we speculate that this might be an experimental artefact due to differences in substrate access $\left(Q_{1}\right)$ to the quinol-binding site of the enzyme in DDM micelles vs the enzyme embedded into the membrane. Importantly, after reconstitution of SMA-cyt $b_{3}$ in $\mathrm{HVs}$, which retains activity of cyt $b o_{3}$, and then re-solubilised 
in DDM detergent, cyt $b_{3}$ shows an activity similar to the DDM-cyt $b o_{3}$ (Figure 4B). This confirms that neither the solubilisation of $\mathrm{cyt} \mathrm{bo}_{3}$ into SMALPs nor the reconstitution into HVs irreversibly changes cyt $\mathrm{bo}_{3}$ (see also Figure S4). In correspondence to the results above, attempting to reconstitute SMA-cyt $b o_{3}$ directly into liposomes was not successful.

\section{A}

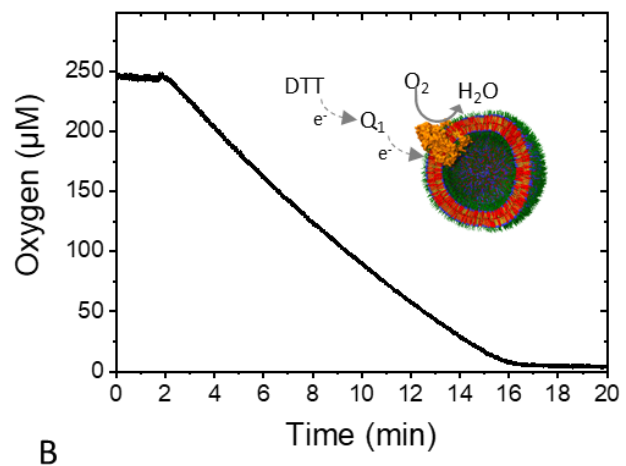

B

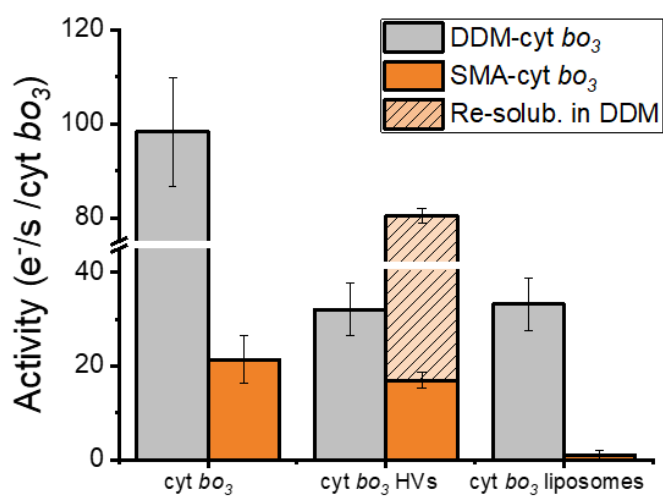

Figure 4 (A) Representative oxygen consumption trace for cyt bo $o_{3}$ activity. The trace shown is for SMAcyt $b_{3} o_{3} \mathrm{HV}$. The oxygen consumption rate was determined via regression of the first 30 seconds from the slope and normalised by the protein concentration. (B) Comparison of the activities of soluble and reconstituted SMA-cyt $b_{3}$ and DDM-cyt bo ${ }_{3}$ determined via oxygen consumption. Error bars represent the standard deviation of three independent experiments.

In order to confirm that reconstituted cyt $b_{3}$ was fully inserted across the membranes of HVs, we evaluated the net change in intravesicular $\mathrm{pH}$ due to the proton-pumping activity of the enzyme upon chemical activation. Changes in internal $\mathrm{pH}$ were determined by ratiometric fluorescence measurements of the $\mathrm{pH}$-sensitive fluorescent probe 8-hydroxypyrene-1,3,6trisulfonic acid (HPTS) (Figure S5, see Supporting Information for details). While HVs showed a constant intravesicular $\mathrm{pH}$ after the addition of DTT and $\mathrm{UQ}_{1}$, both SMA-cyt $b o_{3}$ and DDMcyt $\mathrm{bo}_{3}$ reconstituted $\mathrm{HVs}$ displayed an increase of intravesicular $\mathrm{pH}$ (Figure $5 \mathrm{~A}$ ), similarly to DDM-cyt $b o_{3}$ reconstituted liposomes (Figure 5B). The increase in $\mathrm{pH}$ indicates that the cyt $b o_{3}$ 
was successfully inserted into the membrane with a prevalence of an 'outward' orientation, as previously demonstrated in liposomal reconstitution. ${ }^{[49,50]}$

A

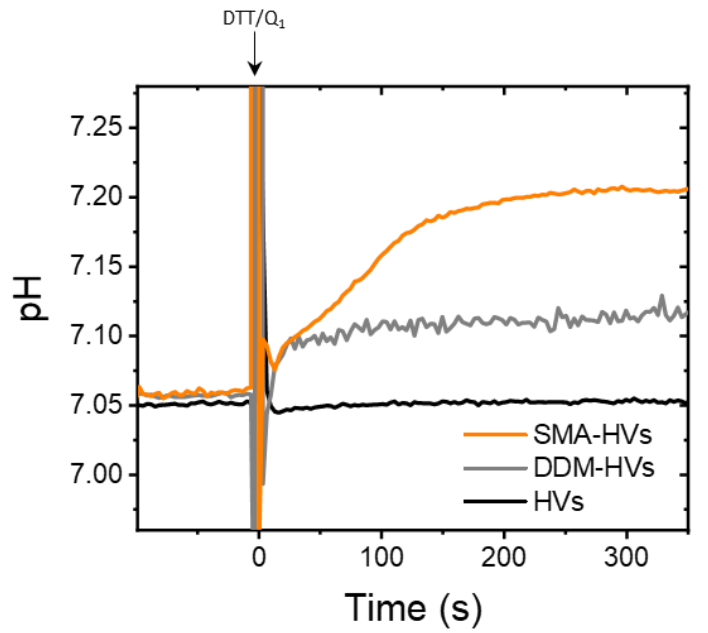

B

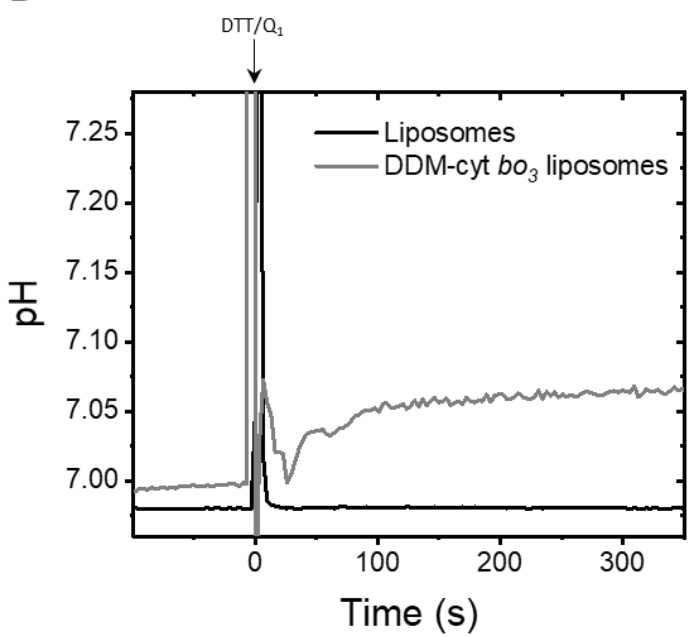

Figure 5 Intravesicular pH change for: (A) HVs, SMA-cyt bo $o_{3}$ reconstituted in HVs (SMA-cyt bo 3 HVs) and DDM-cyt $b o_{3}$ reconstituted in $\mathrm{HVs}$ (DDM-cyt $b o_{3} \mathrm{HVs}$ ), and (B) liposomes and DDM-cyt bo 3 reconstituted in liposome (DDM-cyt bo $o_{3}$ liposomes). Displayed curves are representatives of three independent experiments.

To further assess the ability of SMA to mediate membrane proteins (MPs) reconstitution into $\mathrm{HVs}$, we attempted the reconstitution of the full MPs composition of E. coli. To do this, an $E$. coli membrane extract (GO105/pJRhisA) was solubilised with SMA and non-solubilised material removed by ultracentrifugation $(100,000 \mathrm{~g}$ for $60 \mathrm{~min})$. This full extract of all soluble SMA-MPs was incubated with HVs on ice for $30 \mathrm{~min}$, at a 2:8 protein mass to polymer and lipids mass ratio. SMA-MPs not reconstituted into HVs were again precipitated by addition of $10 \mathrm{mM} \mathrm{MgCl}_{2}$ and removed by centrifugation $(17,000 \mathrm{~g}$ for $15 \mathrm{~min})$. We compared the protein solubilisation efficiencies of soluble and reconstituted SMA-MPs by measuring the protein concentration (bicinchoninic acid (BCA) assay, Table 2). Overall, 52.6 ( \pm 4.6$) \%$ of the $E$. coli MPs were solubilised by SMA. After reconstitution, more than half of this fraction $(29.4$ ( \pm 6.8$) \%)$ was successfully transferred to HVs. 
Table 2 Solubilisation efficiency of $E$. coli membrane proteins extract via soluble and reconstituted SMA-MPs.

\section{Membrane Proteins $\quad$ Solubilisation Efficiency (\%) \pm S.D.}

\begin{tabular}{c|lcc}
\hline & SMA-MPs & 52.6 & 4.6 \\
\multirow{2}{*}{$\begin{array}{c}\text { After } \mathrm{MgCl}_{2} \text { and } \\
\text { centrifugation }\end{array}$} & SMA-MPs (supernatant) & $<1$ & $<1$ \\
& SMA-MPs (pellet) & 43.5 & 8.6 \\
& SMA-MPs HVs & 53.1 & 2.2 \\
After $\mathrm{MgCl}_{2}$ and & SMA-MPs HVs (supernatant) & 29.4 & 6.8 \\
centrifugation & SMA-MPs HVs (pellet) & 21.6 & 5.3 \\
\hline
\end{tabular}

To assess whether the protein content after reconstitution into HVs was a true representation of the various MPs from native membranes of $E$. coli, we conducted an SDS-PAGE analysis for qualitative comparison (Figure S6). SDS-PAGE showed very similar profiles for each condition, strongly suggesting that SMA can extract a wide range of membrane proteins (SMA-MPs) and transfer these to HVs. This analysis also confirmed that precipitation of SMALPs with $10 \mathrm{mM}$ $\mathrm{MgCl}_{2}$ (i.e., without $\mathrm{HVs}$ ) removed the entire protein content. Finally, we evaluated whether the MPs were functionally active after reconstituted into HVs by monitoring the activity of the cyt $b_{3}$, which was part of the MPs extract mixture. Figure 6 and Figure S7 show the oxygen reduction activity of the full MP extracts solubilised by SMA before (SMA-MPs) and after (SMA-MPs HVs) reconstitution into HVs and confirmed that cyt $b_{3}$ was functionally active after transfer into HVs, which suggests that complex mixtures of proteins can be reconstituted with SMA. The oxygen reduction activity, normalized against total MPs content, is lower after reconstitution in HVs and we hypothesize that this is due to different efficiencies of reconstitution of the various MPs. 


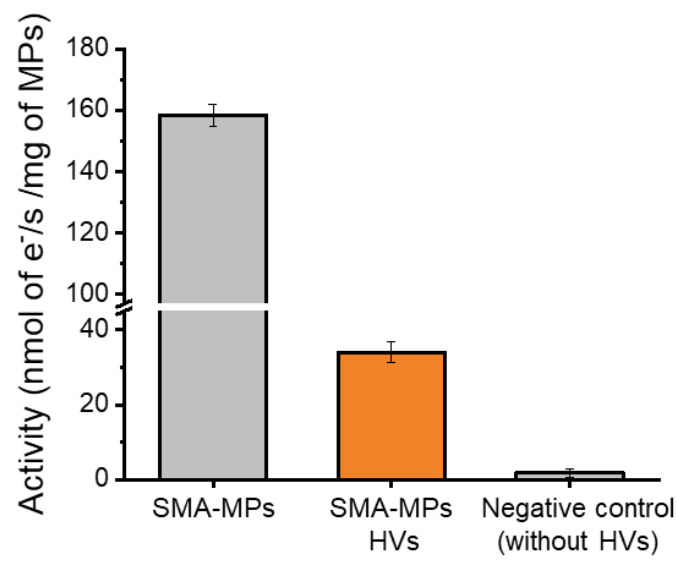

Figure 6 Comparison of the activities of soluble SMA-MPs, reconstituted SMA-MPs in HVs and SMAMPs treated with $\mathrm{MgCl}_{2}$ without $\mathrm{HVs}$ (denoted as 'Negative control') determined via oxygen consumption. The activity is normalised per mg of total MPs content for SMA-MPs and SMA-MPs in $\mathrm{HVs}$ determined via BCA assay. Error bars represent the standard deviation of three independent experiments.

Although SMA-solubilised proteins have previously been shown to reconstitute into planar lipid bilayers ${ }^{[36]}$ or liposomes, ${ }^{[37,38]}$ the mechanisms by which this happens is not fully understood. Indeed, little is known about the interaction between SMALPs and lipid membranes, although it has been shown that the lipid packing properties and electrostatic interactions strongly influence how SMA interplays with the lipid bilayer. ${ }^{[51]}$ Particularly, phospholipid phosphoethanolamine (PE), characterised by a negative intrinsic curvature, ${ }^{[52]}$ exerts a lateral pressure that hampers SMA insertion and, therefore, membrane solubilisation. ${ }^{[51,53,54]}$ Similarly, we hypothesise that PE might hamper SMA reconstitution of MPs back into liposomes. This may explain the lack of reconstitution of SMA-cyt $b o_{3}$ into the liposomes in this study, which were prepared from an E. coli 'polar' lipid extract (PE, 65 mol \%; PG, $25 \mathrm{~mol} \%$ and cardiolipin, $\sim 10 \mathrm{~mol} \%)$. In HVs, the packing of the membrane bilayer is profoundly different from the liposomes, with the amphiphilic block copolymer $\left(\mathrm{PBd}_{22}-\mathrm{PEO}_{14}\right)$ not forming aligned monolayers. ${ }^{[15]}$ Instead, the hydrophobic chains of the block copolymers can become entangled and interdigitate across the two leaflets on the membrane, creating a more disordered (but stable) structure in which MPs can insert. This is in agreement with our destabilisation profiles (Figure S2), which indicate that less SMA is required to solubilise HVs than liposomes. Furthermore, HVs of similar composition have previously been shown to increase permeability to a hydrophobic probe (calcein acetomethoxyl-ester) compared to 
liposomes, indicating less efficient packing properties of the lipid-polymer mixture. ${ }^{[55]}$ Here, we hypothesise that this enhanced disorder of the hybrid membrane allows for the insertion of the MPs.

In conclusion, we have presented a method that allows direct transfer of SMA-solubilised membrane protein, either as pure isolated protein (SMA-cyt bo $o_{3}$ ) or as a complex MPs mixture (SMA-MPs), into HVs without the use of detergents while maintaining protein activity. This provides a new tool to reduce time and cost for enzyme isolation and reconstitution processes by avoiding detergent mediated extraction and represents a solid foundation for further development as an enabling technology for MPs in nanomedicine, biocatalysis and bottomup synthetic biology. 


\section{Acknowledgements:}

We gratefully thank Dr Huijie Zhang for the assistance provided with the setting up of electrochemistry and fluorescence measurements, and Rashmi Seneviratne for training and support on making and characterising hybrid vesicles. This work was funded by the Biotechnology and Biological Sciences Research Council (BBSRC), grant number BB/T000546/1, and the Wellcome Trust, grant number 222373/Z/21/Z.

\section{References}

[1] P. Cintas, Angew. Chemie - Int. Ed. 2020, 132, 7364-7372.

[2] Y. Elani, Angew. Chemie - Int. Ed. 2021, 60, 5602-5611.

[3] G. Wang, K. Castiglione, Catalysts 2019, 9, 1-25.

[4] A. Pannwitz, D. M. Klein, S. Rodrıguez-Jimenez, C. Casadevall, H. Song, E. Reisner, L. Hammarstro, S. Bonnet, Chem. Soc. Rev. 2021, 50, 4833-4855.

[5] H. Yin, A. D. Flynn, Annu. Rev. Biomed. Eng. 2016, 18, 51-76.

[6] L. van der Pol, M. Stork, P. van der Ley, Biotechnol. J. 2015, 10, 1689-1706.

[7] J. Alvarez-Malmagro, G. García-Molina, A. L. De Lacey, Sensors 2020, 20, 1-17.

[8] H. Zhang, R. Catania, L. J. C. Jeuken, Catalysts 2020, 10, 1-29.

[9] E. Rideau, R. Dimova, P. Schwille, F. R. Wurm, K. Landfester, Chem. Soc. Rev. 2018, 47, 8572-8610.

[10] R. Skrzypek, S. Iqbal, R. Callaghan, Methods 2018, 147, 126-141.

[11] A. M. Amati, S. Graf, S. Deutschmann, N. Dolder, C. Von Ballmoos, Biochem. Soc. Trans. 2020, 48, 1473-1492.

[12] D. E. Discher, A. Eisenberg, Science 2002, 967, 967-973.

[13] C. G. Palivan, R. Goers, A. Najer, X. Zhang, A. Car, W. Meier, Chem. Soc. Rev. 2016, 45, 377-411.

[14] C. Lopresti, H. Lomas, M. Massignani, T. Smart, G. Battaglia, J. Mater. Chem. 2009, 19, 
3576-3590.

[15] P. A. Beales, S. Khan, S. P. Muench, L. J. C. Jeuken, Biochem. Soc. Trans. 2017, 45, 1526.

[16] S. Khan, M. Li, S. P. Muench, L. J. C. Jeuken, P. A. Beales, Chem. Commun. 2016, 52, 11020-23.

[17] W. F. Paxton, P. T. McAninch, K. E. Achyuthan, S. H. R. Shin, H. L. Monteith, Colloids Surfaces B Biointerfaces 2017, 159, 268-276.

[18] M. L. Jacobs, M. A. Boyd, N. P. Kamat, Proc. Natl. Acad. Sci. U. S. A. 2019, 116, 40314036.

[19] N. Marušič, L. Otrin, Z. Zhao, R. B. Lira, F. L. Kyrilis, F. Hamdi, P. L. Kastritis, T. Vidaković-Koch, I. Ivanov, K. Sundmacher, R. Dimova, Proc. Natl. Acad. Sci. U. S. A. 2020, 117, 15006-15017.

[20] M. Schulz, W. H. Binder, Macromol. Rapid Commun. 2015, 23, 2031-2041.

[21] J. F. Le Meins, C. Schatz, S. Lecommandoux, O. Sandre, Mater. Today 2013, 16, 397402.

[22] R. Seneviratne, S. Khan, E. Moscrop, M. Rappolt, S. P. Muench, L. J. C. Jeuken, P. A. Beales, Methods 2018, 147, 142-149.

[23] I. Moraes, G. Evans, J. Sanchez-wWatherby, S. Newstead, P. D. Stewart Shaw, Biochim. Biophys. Acta-Biomembr. 2014, 1838, 78-87.

[24] A. M. Seddon, P. Curnow, P. J. Booth, Biochim. Biophys. Acta 2004, 1666, 105-117.

[25] H. Palsdottir, C. Hunte, Biochim. Biophys. Acta 2004, 1666, 2-18.

[26] G. G. Privé, Methods 2007, 41, 388-397.

[27] V. L. G. Postis, S. E. Deacon, P. C. J. Roach, V. L. G. Postis, S. E. Deacon, P. C. J. Roach, G. S. A. Wright, X. Xia, G. S. A. Wright, X. Xia, J. C. Ingram, J. M. Hadden, J. Peter, F. Henderson, S. E. V Phillips, P. C. J. Roach, M. J. Mcpherson, S. A. Baldwin, Mol. Membr. Biol. 2008, 25, 617-624. 
[28] S. C. Lee, T. J. Knowles, V. L. G. Postis, M. Jamshad, R. A. Parslow, Y. Lin, A. Goldman, P. Sridhar, M. Overduin, S. P. Muench, T. R. Dafforn, Nat. Protoc. 2016, 11, 11491162.

[29] T. J. Knowles, R. Finka, C. Smith, Y. Lin, T. Dafforn, M. Overduin, J. Am. Chem. Soc. Commun. 2009, 131, 7484-7485.

[30] M. Jamshad, J. Charlton, Y. Lin, S. J. Routledge, Z. Bawa, T. J. Knowles, M. Overduin, N. Dekker, T. R. Dafforn, R. M. Bill, D. R. Poyner, M. Wheatley, Biosci. Rep. 2015, 35, e00188.

[31] S. R. Tonge, B. J. Tighe, Adv. Drug Deliv. Rev. 2001, 53, 109-122.

[32] M. Esmaili, M. Overduin, Biochim. Biophys. Acta-Biomembr. 2018, 1860, 257-263.

[33] P. S. Orekhov, M. E. Bozdaganyan, N. Voskoboynikova, A. Y. Mulkidjanian, H.-J. Steinhoff, K. V Shaitan, Langmuir 2019, 35, 3748-58.

[34] S. J. Hesketh, D. P. Klebl, A. J. Higgins, M. Thomsen, I. B. Pickles, F. Sobott, A. Sivaprasadarao, V. L. G. Postis, S. P. Muench, Biochim. Biophys. Acta-Biomembr. 2020, 1862, 183192.

[35] N. L. Pollock, S. C. Lee, J. H. Patel, A. A. Gulamhussein, A. J. Rothnie, Biochim. Biophys. Acta-Biomembr. 2018, 1860, 809-817.

[36] J. M. Dörr, M. C. Koorengevel, M. Schäfer, A. V Prokofyev, S. Scheidelaar, E. A. W. van der Cruijesn, T. R. Dafforn, M. Baldus, A. J. Killian, Proc. Natl. Acad. Sci. U. S. A. 2014, $111,18607-18612$.

[37] I. A. Smirnova, P. Ädelroth, P. Brzezinski, Sci. Rep. 2018, 8, 14950.

[38] D. Dutta, M. Esmaili, M. Overduin, L. Fliegel, Biochim. Biophys. Acta-Biomembr. 2020, 1862, 183111.

[39] A. O. Oluwole, B. Danielczak, A. Meister, J. O. Babalola, C. Vargas, S. Keller, Angew. Chemie - Int. Ed. 2017, 56, 1919-1924.

[40] A. A. Gulamhussein, R. Uddin, B. J. Tighe, D. R. Poyner, A. J. Rothnie, Biochim. Biophys. Acta-Biomembr. 2020, 1862, 183281. 
[41] M. Barniol-Xicota, S. H. L. Verhelst, Commun. Biol. 2021, 4, 218.

[42] G. M. Di Mauro, C. La Rosa, M. Condorelli, A. Ramamoorthy, Langmuir 2021, 37, 3113-3121.

[43] S. C. L. Hall, C. Tognoloni, J. Charlton, É. C. Bragginton, A. J. Rothnie, P. Sridhar, M. Wheatley, T. J. Knowles, T. Arnold, K. J. Edler, T. R. Dafforn, Nanoscale 2018, 10, 10609-10619.

[44] A. J. Garcia-Horsman, B. Barquera, J. Rumbley, J. Ma, R. B. Gennis, J. Bacteriol. 1994, $176,5587-5600$.

[45] S. K. Lim, H.-P. de Hoog, A. N. Parikh, M. Nallani, B. Liedberg, Polymers 2013, 5, 11021114.

[46] J. N. Rumbley, E. F. Nickels, R. B. Gennis, Biochim. Biophys. Acta - Protein Struct. Mol. Enzymol. 1997, 1340, 131-142.

[47] K. A. Morrison, A. Akram, A. Mathews, Z. A. Khan, J. H. Patel, C. Zhou, D. J. Hardy, C. Moore-kelly, R. Patel, V. Odiba, T. J. Knowles, M.-H. Javed, N. P. Chmel, T. R. Dafforn, A. J. Rothnie, Biochem. J. 2016, 473, 4349-4360.

[48] Y. Liu, E. C. C. M. Moura, J. M. Dörr, S. Scheidelaar, H. Heger, M. R. Egmond, J. A. Killian, T. Mohammadi, E. Breukink, PLoS One 2018, 13, 1-18.

[49] M. Li, S. K. Jørgensen, D. G. G. Mcmillan, Ł. K. Krzemiński, N. N. Daskalakis, R. H. Partanen, M. Tutkus, R. Tuma, D. Stamou, N. S. Hatzakis, L. J. C. Jeuken, J. Am. Chem. Soc. 2015, 137, 16055-16063.

[50] I. Mazurenko, N. S. Hatzakis, L. J. C. Jeuken, J. Vis. Exp. 2019, 1-11.

[51] S. Scheidelaar, M. C. Koorengevel, J. D. Pardo, J. D. Meeldijk, E. Breukink, J. A. Killian, Biophys. J. 2015, 108, 279-290.

[52] B. Kollmitzer, P. Heftberger, M. Rappolt, G. Pabst, Soft Matter 2013, 9, 10877-10884.

[53] J. M. Dörr, S. Scheidelaar, M. C. Koorengevel, J. J. Dominguez, M. Schäfer, C. A. van Walree, J. A. Killian, Eur. Biophys. J. 2016, 45, 3-21.

[54] J. J. Dominguez Pardo, J. M. Dörr, A. Iyer, R. C. Cox, S. Scheidelaar, M. C. Koorengevel, 
V. Subramaniam, J. A. Killian, Eur. Biophys. J. 2017, 46, 91-101.

[55] S. Rottet, S. Iqbal, P. A. Beales, A. Lin, J. Lee, M. Rug, C. Scott, R. Callaghan, Polymers 2020, 12, 1049. 
